\title{
Wireless Sensor Networks Applications via High Altitude Systems
}

\author{
Zhe Yang and Abbas Mohammed \\ Blekinge Institute of Technology \\ Sweden
}

\section{Introduction}

Wireless sensor networking is a fast emerging subfield in the field of wireless networking. It is a key technology for the future ad has been identified as one of the most important technologies for this century (Akyildiz et al., 2002; Business Week, 1999; Technology Review, 2003). These sensors are generally equipped with data processing, communication, and information collecting capabilities. They can detect the variation of ambient conditions in the environment surrounding the sensors and transform them into electric signal (e.g., temperature, sound, image). Interests in sensor networks have motivated intensive research in the past few years emphasizing the potential of collaboration among sensors in data collecting and processing, coordination and management of the sensing activity and date flow to the sink.

Depending on application to reveal some characteristics about phenomena in the area, sensor nodes can be deployed on the ground, in the air, under water, on bodies, in vehicles and inside buildings (Akyildiz et al., 2002). Thus, these connected sensor nodes have many promising applications in many fields (e.g., consumer, military, health, environment, security). Deployment of these sensor nodes can be in random fashion like dropping from a helicopter (a disaster management setup), or manual (deploying nodes in a building to detect the movement of human) (Akyildiz et al., 2002).

Sensor nodes are usually constrained in energy and bandwidth (Akyildiz et al., 2002). Such constraints combined with the deployment of a large number of sensor nodes are challenges to the design and maintenance of sensor networks. Energy-awareness has to be considered at all layers of networking protocol stack. It is also related to physical and link layers which are generally common for all kind of sensor applications. Research on these layers has been focused on radio communication hardware, energy-aware media access control (MAC) protocols (Demirkol et al., 2006; Hill et al., 2000; Intel, 2004; Jiang et al., 2006). The main aim at the network layer is to find ways for energy-efficient and reliable route setup from sensor nodes to the sink in order to maximally extend the lifetime of network.

HAPs are either aircraft or airships operating at an altitude of $17 \mathrm{~km}$ above the ground. They have been suggested by the International Telecommunication Union (ITU) for providing communications in mm-wave broadband wireless access (BWA) and the third generation (3G) communication frequency bands (Elabdin et al., 2006; Thornton et al., 2003; 
Tozer \& Grace, 2001). Currently, investigations on HAPs have been carried on in the 3G telecommunication and broadband wireless services. These platforms are regarded to be based on lighter-than-air vehicles or conventional aircraft proposed at various stages of development (Tozer \& Grace, 2001). Employing unpiloted, solar-powered platforms in different altitudes can ultimately make the systems more reliable and competitive in the future.

HAP systems have many characteristics to make it competitive to be adopted in different telecommunication and wireless communication applications, e.g. a mobile sink in WSN. HAPs can provide high receiver elevation angle, line of sight (LOS) transmission, large coverage area and mobile deployment etc. The system combines the advantages of terrestrial and satellite systems, and furthermore contributes to a better overall system performance, greater system capacity and cost-effective deployment (Mohammed et al., 2008). Many countries have made significant efforts in the research of HAP systems and their potential applications. A company Strat $X X^{\circledR}$ in Switzerland has started to develop three different platforms operating from $3 \mathrm{~km}$ to $17 \mathrm{~km}$ above the ground to provide various services, e.g. mobile multimedia transmission, local navigation and remote sensing (Strat XX, 2008). A similar scenario of using unmanned autonomous vehicle (UAV) to transfer information in the distributed wireless sensor system has been proposed (Vincent et al., 2006) and shown to be an energy-efficient solution.

In this chapter, we explore and analyze the potential of using HAPs in WSN applications to establish a HAP-WSN system. The HAP-WSN system is composed of a large number of sensor nodes, which can monitor and collect information about the physical environment and transmit the data to another location for processing in an ad-hoc manner, and a HAP, which collects information from sensor nodes as a remote sink above the ground. Reliable communication links are analyzed between sensor nodes and HAPs to achieve LOS in most cases based on the height of the platform. The HAP-WSN can be deployed in inaccessible or disaster environments, where sensor nodes and HAPs are both powered by battery, which means energy consumption is the key concept in the system design. The chapter is organized as follows: in section 2, an introduction to WSN and HAP-WSN system is given. Two scenarios of HAP-WSN are proposed based on the cell formation of the HAP system and sensor node radio link. In section 3, the configuration and simulation results in the system level of HAP-WSN are presented. In section 4, the configuration and simulation results in the physical layer are presented. In section 5, conclusions and future research are given.

\section{High Altitude Platform-Wireless Sensor Network System}

\subsection{WSN communication scenarios and design issues}

A typical sensor network contains a large number of sensor nodes with data processing and communication capabilities. The sensor nodes send collected data via radio transmitter, to a sink either directly or through other nodes in a multi-hop fashion. The technological advances in this field result in the decrease of the size and cost of sensors and enabled the development of smart disposable micro sensors, which can be networked through wireless links. Fig. 1 shows the communication architecture of a WSN. Sensor nodes organize themselves to collect highly reliable information about the phenomenon, and route data via other sensors to the sink. The sink in Fig. 1 could be either a fixed or mobile node with the 
capability of connecting sensor networks to the outer existing communication infrastructure, e.g. internet, cellular and satellite networks.

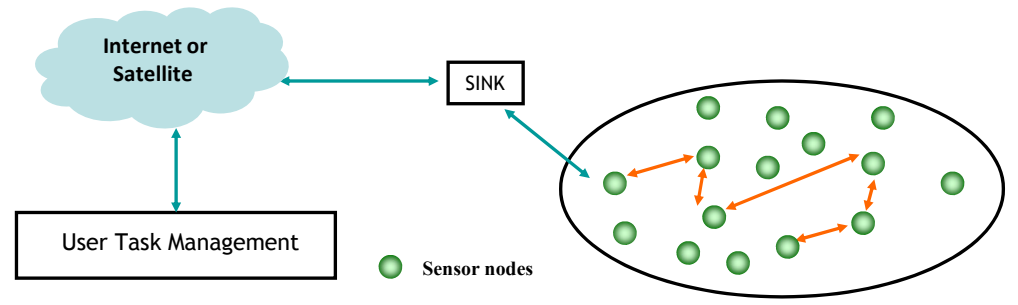

Fig. 1. General communication scenarios of a WSN

Due to the number of sensor nodes and the dynamics of their operating environment, it poses unique challenges in the design of sensor network architecture.

Dynamic network: Basically a WSN consists of three components: sensor node, sink and event. Sensor nodes and sink are assumed to be fixed and mobile. Although currently sensor nodes in most applications are assumed to be stationary, it is still necessary to support the mobility of sinks or gateway in the network. Thus the stability of data transferring is an important design factor, in addition to energy, bandwidth etc (Akyildiz et al., 2002). Moreover the phenomenon could also be dynamic, which requires periodic report to the sink.

- Energy constrains: The process of data routing in the network is greatly affected by energy considerations, routing path and radio link. Since the radio transmission in practical scenarios degrades with distance much faster than transmission in free space, means that communication distance and energy must be well managed (Chong \& Kumar, 2003). Directed routing would perform well enough if all the sensor nodes are close to the sink. However, most of the time, it is necessary to use multi-hop routing to consume less power than directed routing, since sensors are randomly scattered in the area.

- Propagation environment: Sensor nodes are deployed on the ground which leads to a relative low height of antenna on a sensor node and a small distance to the radio horizon. Non line of sight (NLOS) signal transmission in WSN is predominant in most directions since the complicated environment of deployment can cause severe attenuations. Signal power at a distance $d$ away from the transmitter may be estimated as $1 / d^{n}$, where $n=2$ for propagation in free space, but $n$ is between 2 and 4 for low lying antenna deployments in practical WSNs (Vincent et al., 2006).

There are other issues such as coverage area, scalability, transmission media, routing protocols, which could also affect the design and performance of the network (Akyildiz et al., 2002; Chong \& Kumar, 2003). All the solutions to these issues need to reduce the energyconsumption and prolong the lifetime of WSN in most applications.

\subsection{HAP-WSN System Scenarios and Advantages}

Current research in HAPs has widely adopted two proposed types of cell planning in HAP system. By subdividing the coverage area of the HAP into one or multiple cells, the HAP 
antenna payload has potential to provide a high gain in each cell planning scenario. In (Thornton et al., 2003; Yang et al., 2007), the coverage area has been divided into 121 and 19 cells in order to improve the capacity of HAP system. Based on the architecture of HAPs and WSN, we propose two configurations for HAP-WSN systems for different applications. The first scenario is shown in Fig. 2. The sensor nodes inside the HAP cells are transmitting information directly to the HAP. The main aim of the scenario is to reduce the complexity and remove energy-consumption of multi-hop transmissions in WSN. It is suitable for WSN applications with low data transmission in large coverage area.

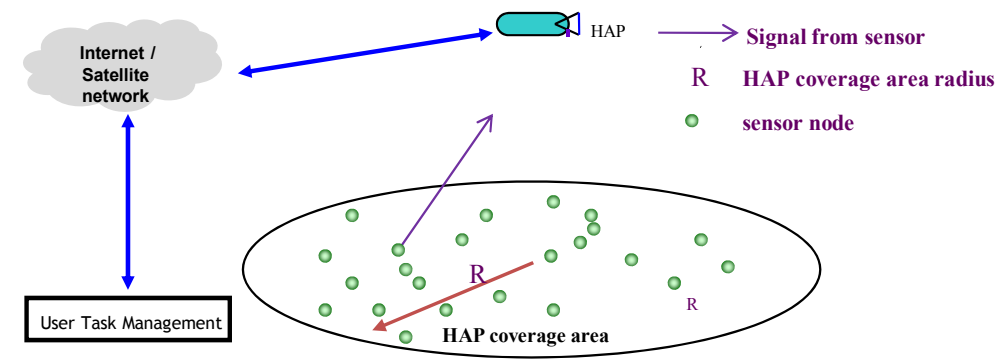

Fig. 2. A HAP-WSN system in a single cell configuration.

Fig. 3 shows the second system configuration of the HAP-WSN. The sensor nodes inside the HAP cell are organized into a cluster, where one node with the higher-energy is selected as the cluster head. Senor nodes as cluster members collect information and send to the cluster head, which is responsible to send all data to the HAP. The cluster formation in WSNs is typically based on the energy reserve of sensors and their distances to the cluster head (Akyildiz et al., 2002). The main aim of the scenario is to reduce the complexity of a multihop WSN and maintain the energy consumption of all sensor nodes. It can be employed in WSN applications with high data transmission requirement, e.g. multimedia.

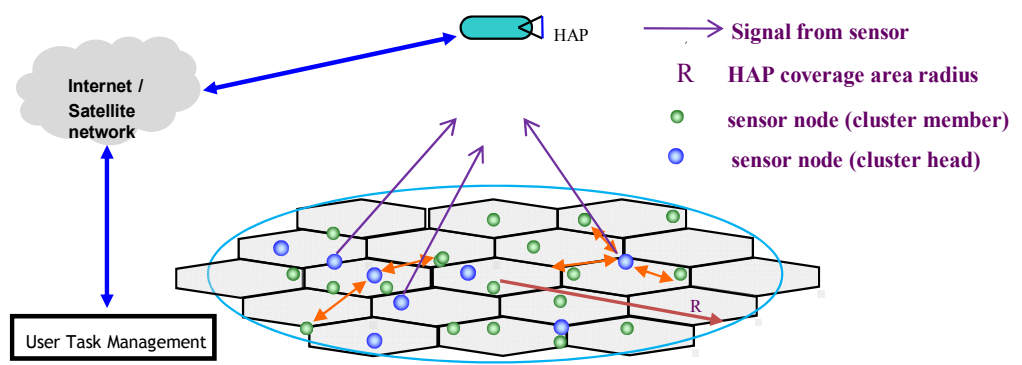

Fig. 3. A HAP-WSN system in a multi-cell configuration

The HAP-WSN system has advantages of HAP system which is employed as a sink in the WSN:

- Reducing complexity of multi-hop transmission and achieving energy-efficiency: A multi-hop routing has been under investigations because the radio link is usually constrained by obstructions on the ground. HAPs are often considered to be located a few kilometers above the ground, where it can establish a LOS link 
between the sensor node and the HAP sink. Therefore HAPs offer a potential of reducing or removing transmission burden in WSN, organize communications based multiple access schemes, e.g. TDMA, CDMA, to reduce energy consumption in sensor nodes.

- Low cost and rapid mobile deployment: It is believed that the cost of HAP is considerably cheaper than that of a satellite because HAPs do not require expensive launch and maintenance (Tozer \& Grace, 2001). The HAP as a sink, can be reused, repaired and replaced quickly for applications of WSNs, e.g. disaster and emergency surveillance where it has clear advantages. It may stay in the sky for a long period, which can prolong the life of the WSN.

\section{System Level Configuration and Simulation Performance}

\subsection{HAP system antenna and propagation issues}

In this work we employ a directive antenna payload on HAPs, which can ensure more power radiated in the desired directions. The HAP antenna payload is assumed to be composed of either a single or multiple antennas according to the cell formation. The antenna radiation model is presented in (Thornton et al., 2003). The gain of the antenna of $\mathrm{HAP} A_{H}(\varphi)$, at an angle $\varphi$ with respect to its boresight, is approximated by a cosine function raised to a power roll-off factor $n$ and a notional flat sidelobe level $S_{f}$. $G_{H}$ represents the boresight gain of the HAP antenna.

$$
A_{H}(\varphi)=G_{H}\left(\max \left[\cos (\varphi)^{n_{H}}, s_{f}\right]\right)
$$

The antenna peak gain is accordingly achieved at the centre of the HAP cell. The HAP antenna beamwidth is initially defined by its $\varphi_{10 d B}$ set to be equal to the subtended angle away from the antenna boresight of the central cell to the edge of the HAP coverage area or the central HAP cell corresponding to the single and multi-cell formations. After defining the beamwidth, the boresight gain is calculated as (Thornton et al., 2003):

$$
G_{\text {boresight }}=\frac{32 \ln 2}{2 \theta^{2}{ }_{3 d B}}
$$

We select the roll-off factor $n$ to let the radiation curve falling to $10 \mathrm{~dB}$ lower than the maximum value. Fig. 4 shows the two HAP antenna radiation masks corresponding to the single or multiple cell structures in the system. 

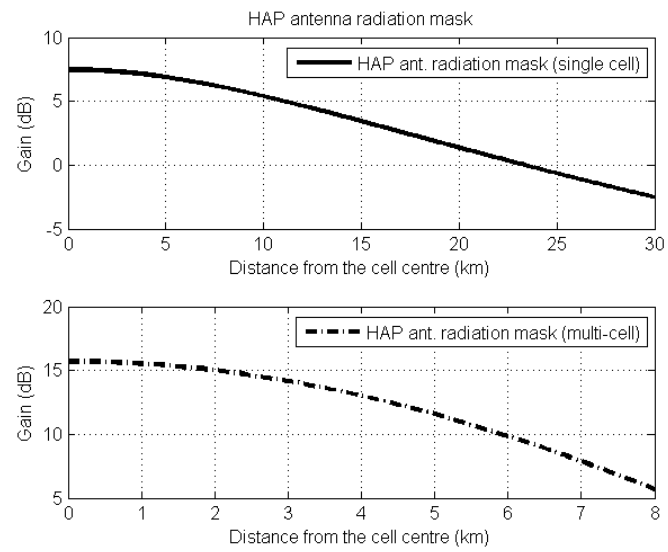

Fig. 4. HAP antenna radiation masks in a single cell and multi-cell formation.

Distance attenuation is the empirically observed long-term trend in signal loss as a function distance, which is typically proportional to the range raised to some power. A shadowing fading is used to represent the shadowing effect, which considers the surrounding environmental clutter that may be different at two locations with the same separation distance. In our scenario, the pathloss between HAP and sensor node is expressed as the log-distance pathloss and log-normal shadowing model:

$$
P L(d)[d B]=\overline{P L}\left(d_{0}\right)[d B]+10 n \log \left(\frac{d}{d_{0}}\right)+X_{\sigma}
$$

where $n$ is the pathloss exponent, $d_{0}$ is the reference distance and $d$ is the separation distance between HAP and sensor node. The value of $n$ is between 2 and 6 depending on the propagation environment. $X \sigma$ denotes a zero mean Gaussian random variable with a standard deviation $\sigma$ (in $\mathrm{dB}$ ). The model shows that the pathloss at the particular location is random and log-normally distributed about the mean distance dependent value.

\subsection{System evaluation criteria and parameters}

Considering a sensor node in the location $(x, y)$ to communicate with the HAP, performance can be evaluated by energy bit to noise spectral density ratio in (4):

$$
\frac{E_{b}}{N_{0}}(x, y)=\frac{P_{s} A_{s} A_{H} P L_{S H}}{N_{0} R_{b}}
$$

where,

$P_{s}$ is the transmission power of a sensor node in the target HAP cell.

$A_{s}$ and $A_{u}$ are antenna gains of a sensor node and HAP respectively.

$P L_{S H}$ is the signal pathloss due to distance attenuation and shadowing effect depending on the location of sensor node. 
$R_{b}$ is the data rate of senor node.

$N_{0}$ is the noise power spectral density.

Evaluation parameters are shown in Table 1. The physical later (PHY) parameters, e.g. data rate, sensor node transmit power, are referred to product data sheets of the company Crossbow ${ }^{\circledR}$ specializing on the sensor network technology (Crossbow, 2008). Parameters of the low speed $\left(R_{b}=38.4 \mathrm{kbps}\right)$ and high speed $\left(R_{b}=250 \mathrm{kbps}\right)$ senor nodes are referred for different applications.

Table 1. System level simulation parameters

\begin{tabular}{|l|l|}
\hline Parameters & Settings \\
\hline $\begin{array}{l}\text { Data Rate }\left(\mathrm{R}_{\mathrm{b}}\right) \\
\text { Tx Power }\left(\mathrm{P}_{\mathrm{s}}\right)\end{array}$ & $250 \mathrm{kbps} / 38.4 \mathrm{kbps}$ \\
Tx Antenna Gain Rx $\left(\mathrm{A}_{\mathrm{s}}\right)$ & $3 \mathrm{dBm} / 5 \mathrm{dBm}$ \\
& 1 \\
\hline HAP Antenna Boresight $\left(\mathrm{G}_{\mathrm{H}}\right)$ & $7 \mathrm{~dB} / 16 \mathrm{~dB}$ \\
HAP Height & $17 \mathrm{~km}$ (typical) \\
Coverage Radius $(\mathrm{R})$ & $30 \mathrm{~km}($ typical $)$ \\
Cell Radius & $30 \mathrm{~km} / 8 \mathrm{~km}($ multi-cell $)$ \\
\hline Pathloss Exponent $(\mathrm{n})$ & 2 \\
Propagation Model & Free space \\
Shadowing Std. Deviation $(\sigma)$ & $2 \mathrm{~dB}(\mathrm{Log}-\mathrm{normal})$ \\
ISM Frequency Band & $2.4 \mathrm{GHz} / 868 \mathrm{MHz}$ \\
Noise Power Spectral Density $\left(\mathrm{N}_{0}\right)$ & $3.98 \mathrm{e}-21 \mathrm{~W} / \mathrm{Hz}$ \\
\hline
\end{tabular}

\subsection{System level evaluation results}

The cumulative distribution function $(\mathrm{CDF})$ of $E_{b} / N_{0}$ is used to evaluate the system performance. Fig. 5 shows the $C D F$ of $E_{b} / N_{0}$ of the received signal in single cell and multi cell scenario with different transmission rate. According to the product data sheet in (Crossbow, 2008), industrial-scientific-medical (ISM) band at $868 \mathrm{MHz}$ and $2.4 \mathrm{GHz}$ is selected, respectively. It can be seen that transmission from sensor node to HAP at $17 \mathrm{~km}$ in two scenarios is possible under the coverage area of $30 \mathrm{~km}$ in radius. The performance of sensors in multi cell scenario is enhanced compared to the single cell HAP-WSN system with the same transmission rate due to improved HAP cellular antenna radiation profile.

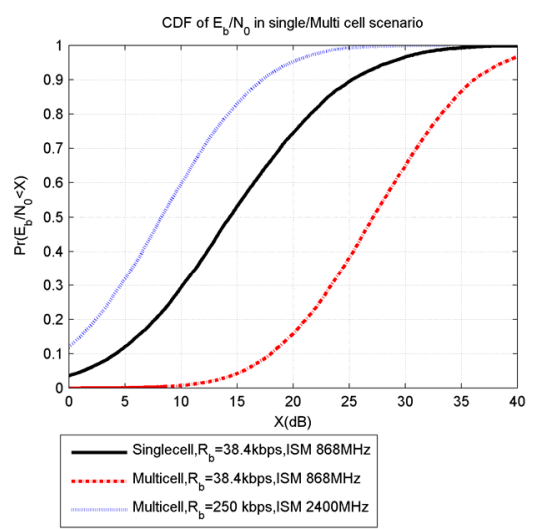

Fig. $5 . \mathrm{E}_{\mathrm{b}} / \mathrm{N}_{0}$ of sensor node with different transmission rate in the single cell and multi cell HAP-WSN scenario 


\section{Physical Layer Configuration and Simulation}

Reliable communication links are needed to be established between sensor nodes and HAPs to achieve a LOS in most cases based on the height of the platform. Our investigations in section 3 show the possibility of establishing a radio link between HAPs and sensor nodes. In this section, we investigate the performance of the promising multiple access scheme based on OFDM in conjunction with the HAP served as a mobile sink to communicate with multiple sensor nodes.

\subsection{Time-varying HAP channel characteristics}

The HAP communications channel exhibits time-varying characteristics due to the motion of the platform or receivers and frequency selectivity due to the multipath propagation. Doppler spectrum can be used to characterize a fading channel and determine if the fading is fast or slow. A simpler parameter, the maximum Doppler spread $f_{m}$, can be used to determine the channel coherence time $T_{c}$ as (Rappaport, 1996):

$$
T_{c}=\frac{9}{16 \pi f_{m}}
$$

where the maximum Doppler spread $f_{m}$ at the carrier frequency $f_{o}$ is:

$$
f_{m}=f_{d, H A P}+f_{d, \text { sensor }}=\left[v_{\text {HAP }}+v_{\text {sensor }}\right] \frac{f_{0}}{c}
$$

where $v_{\text {HAP }}$ and $v_{\text {sensor }}$ is the speed of HAP and sensor node, respectively. According to (Papathanassiou et al., 2001), the Doppler shift exhibits a well-behaved and rather deterministic variation with time. If we assume the HAP station is not moving, the multipath signals arriving at the HAP demonstrate unequal but relative small Doppler shifts, which illustrates that the second Doppler spread component exhibits a relatively small value and can be modeled in accordance to the typical techniques employed in terrestrial mobile radio system (Palma-Lazgare \& Delgado-Penin, 2006; Papathanassiou et al., 2001).

In HAP-WSN applications, sensor nodes are mostly not capable of mobility and thus we don't take account of the movement of sensor nodes. It is one of advantages of using aerial platform compared to UAV since platforms can be more stably deployed upon the area of interest with a long duration.

The selectivity of channel is evaluated by the coherence bandwidth $B_{c}$ of the channel, where $B_{c}$ is approximately equal to the inverse of the maximum delay spread $\tau_{m}$. In time domain, if the bandwidth of a signal is larger than the reciprocal of the maximum delay spread $\tau_{\mathrm{m}}$, each multipath signal can be modelled separately since different paths are resolvable. For a typical LEO channel, the $\tau_{m}$ ranges from 250 to 800 ns (Papathanassiou et al., 2001). Due to similarities of HAP and LEO satellites, we model the HAP channel as a slow-varying and frequency-selective fading channel. We assume the HAP is relatively stationary, thus the Doppler shift due to the motion of the HAP is assumed to be eliminated. The channel is 
regarded to be a quasi-stationary, and so the fading profile can be regarded to be invariant during the period of one symbol.

The HAP channel is modelled as an impulse channel response $h(t)$ with a sequence of discrete-time complex valued components. This sequence of discrete-time complex valued taps of a channel can be generally expressed by the vector $h$, which is equal to $\left[h_{1} h_{2} \ldots h_{l}\right]$, where $l$ is the length of discrete-time channel length, and $h_{l}$ is the complex value of the $l^{\text {th }}$ tap. HAP channel modelling parameters are listed in Table 2.

Table 2. HAP channel characteristics

\begin{tabular}{|l|l|}
\hline HAP Speed $\left(v_{\text {HAP }}\right)$ & stationary \\
\hline Node Speed $\left(v_{\text {sensor }}\right)$ & stationary \\
\hline System bandwidth $(B)$ & $5 \mathrm{MHz}$ \\
\hline Carrier Frequency & ISM band $2.4 \mathrm{GHz}$ \\
\hline Channel Model & $\begin{array}{l}\text { Time-Flat } \\
\text { Frequency-Selective }\end{array}$ \\
\hline Max delay spread $\left(\tau_{m}\right)$ & $500 \mathrm{~ns}$ \\
\hline Power delay profile & exponential with $\tau_{m}$ \\
\hline Fading & $\begin{array}{l}\text { Ricean } \\
\text { Rayleigh }\end{array}$ \\
\hline
\end{tabular}

\subsection{Multiple access schemes of OFDM}

Orthogonal frequency-division multiplexing/Time division multiple access (OFDM/TDMA) is based on OFDM transmission scheme and time-division multiple access. Usually the overall bandwidth in OFDM/TDMA is divided into $N$ subcarriers, and each subcarrier is carrying relatively small signalling rate. It has to be noticed that a precise synchronization between sensor nodes and HAP is required in order to have the flexibility and multiple node accessing. Furthermore the situation leads to a high implementation complexity both in sensor nodes and HAP. In this chapter, we consider a light version of OFDM/TDMA, where a single sensor node uses a full time slot to transmit, and the data rate stream is split into a number of low rate signals modulated in each subcarrier.

Consider the equation for the baseband complex signal of one OFDM symbol in the discretetime domain:

$$
x_{\text {data }}(n)=\sum_{k=0}^{N-1} X_{k} \exp \left(j \frac{2 \pi}{N} k n\right) \quad \mathrm{n} \in(0,1,2, \cdots, \mathrm{N}-1)
$$

We use $N$-long vector $X_{\text {data }}$ to denote the total OFDM data to be part of the IFFT output:

$$
X_{\text {data }}=\left[x_{\text {data }, 1}, x_{\text {data }, 2}, \ldots, x_{\text {data }, N}\right]
$$

Furthermore, let $X_{G I}$ be an $N_{G I}$-long vector expressing the guard interval (GI) precursor signal of $X_{\text {data }}$. $X_{G I}$ is chosen to be equal the last $N_{G I}$ elements of $X_{\text {data }}$, and is denoted as cyclic prefix $(\mathrm{CP})$. So a completed transmitted OFDM symbol is given by: 


$$
X=\left[X_{G I} X_{\text {data }}\right]
$$

Adjacent orthogonal subcarrier frequency separation $B_{\text {sub }}$ is equal to $B / N$, and is chosen to let each subcarrier experience a favourable frequency non-selective fading based on $N$. Usually $N$ is chosen to make the minimum coherence bandwidth $B_{c}$, which is approximately equal to the inverse of the maximum delay spread $\tau_{m}, 10$ times higher than the $B_{\text {sub }}$ (Papathanassiou et al., 2001).

$$
B_{\text {sub }}=(B / N)<\frac{B_{c}}{10} \approx \frac{1}{10 \tau_{m}}
$$

\subsection{Simulation setup and results}

For a HAP channel at a carrier frequency of $2.4 \mathrm{GHz}$ with $\tau_{m}$ equal to $500 \mathrm{~ns}$, the minimum coherence bandwidth is equal to $2 \mathrm{MHz}$. Therefore, if we choose $N$ equal to 64 , the bandwidth of an individual carrier frequency is equal to $78.125 \mathrm{kHz}$. Each subcarrier can be guaranteed to be nonselective. In order to keep the orthogonality of the OFDM symbol, CP is inserted and the $\mathrm{N}_{G I}$ is equal to 3. Therefore, the duration of $\mathrm{CP}$ is equal to $0.6 \mathrm{~ms}$, which is larger than the $\tau_{m}$. In an individual OFDM symbol, CP occupies 4.4 percentage of the symbol $X$ and can be regarded to be high-efficient transmission. The channel estimation is performed base on pilot symbols with a data interval at 8 in one OFDM symbol (Cai \& Giannakis, 2004). In order to reduce the complexity of the problem, we have adopted a simplified but valuable approach purely based on BER performance, which can be achieved by a single sensor node. In other words, multiple sensor transmission scenario is not considered in our simulations since it usually requires a precise synchronization when a large number of sensor nodes transmitting at the same time. No coding schemes are considered in the simulation. Binary phase-shift keying (BPSK) is used to modulate sensor node data rate $R_{b}$ at $250 \mathrm{kbps}$. The system is assumed to be perfectly synchronized.

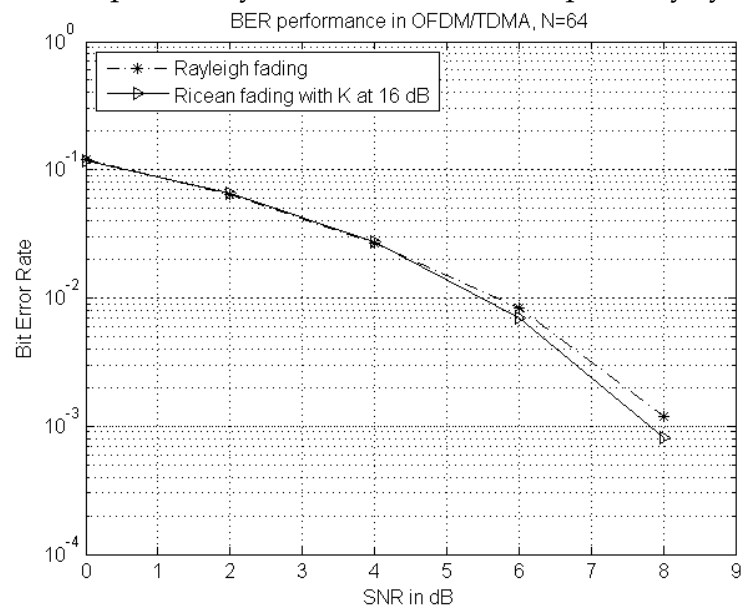

Fig. 6. BER performance of OFDM/TDMA in HAP-WSN 
Simulation results in Fig. 6 show the bit error rate (BER) for $\mathrm{N}$ at 64 . Generally, multipath can degrade the system performance due to severe signal attenuation. However, one of the main advantages of OFDM scheme is its improved performance and robustness in multipath environments, which is predominant in signal transmission of WSN. Consequently, it can be seen from Fig. 6 that there is a little difference in the BER performance under Rayleigh and Ricean fading in the investigated scenario.

\section{Conclusion and Future Research}

In this chapter, we have shown the scenarios of using HAP as a sink in the WSN in ISM band for different data rate transmission and examined the performance in the system level and physical layer. The HAP-WSN system can reduce complexity of the WSN and prolong the lifetime of sensor node by effectively decreasing or removing the multi-hop transmission. The HAP-WSN has a great potential in extending coverage area of WSN due to the unique height of the HAP. A LOS free space pathloss and log-normal shadowing model has been employed to examine the radio link between HAP and sensor nodes. It can be seen that employing HAP as a sink is possible and a promising application of WSN. In future work, a study of multiple access scheme based CDMA for HAP-WSN is promising. Furthermore, a comparison study of multiple access techniques based on OFDMA and CDMA using comparable system parameters can also be investigated to show the advantages of each scheme.

\section{References}

Akyildiz, I. F., Weilian Su, Sankarasubramaniam, Y., \& Cayirci, E. (2002). A Survey on Sensor Network. IEEE Communications Magazine, Vol. 40, No. 8, August 2002, $102-$ 114.

Business Week. (1999, August 30). 21 Ideas for the 21st Century. Business Week, 78-167.

Cai, X., \& Giannakis, G. B. (2004). Error Probability Minimizing Pilots for OFDM with MPSK Modulation over Rayleigh Fading Channels. IEEE Transactions on Vehicular Technology, 53(1), 146-155.

Chong, C.-Y., \& Kumar, S. P. (2003). Sensor Networks: Evolution, Opportunities, and Challenges. Proceedings of the IEEE, 91.

Crossbow. (2008). Product Reference Guide. from http://www.lindstrand.co.uk

Demirkol, I., Ersoy, C., \& Alagöz, F. (2006). MAC Protocols for Wireless Sensor Networks: A Survey. IEEE Communications Magazine

Elabdin, Z., Elshaikh, O., Islam, R., Ismail, A. P., \& Khalifa, O. O. (2006). High Altitude Platform for Wireless Communications and Other Services. International Conference on Electrical and Computer Engineering, 2006, ICECE '06

Hill, J., Szewczyk, R., Woo, A., Hollar, S., E.Culler, D., \& Pister, K. S. J. (2000). System Architecture Directions for Networked Sensors. In Architectural Support for Programming Languages and Operations Systems, 93-104.

Intel. (2004). Instrumenting the Word-An introduction to Wireless Sensor Networks.

Jiang, P., Wen, Y., Wang, J., Shen, X., \& Xue, A. (2006, June 21-23). A Study of Routing protocols in Wireless Sensor Networks. 6th World Congress On Intelligent Control and Automation, Dalian, China. 
Mohammed, A., Arnon, S., Grace, D., Mondin, M., \& Miura, R. (2008). Advanced Communications Techniques and Applications for High-Altitude Platforms. Editorial for a Special Issue, EURASIP Journal on Wireless Communications and Networking, 2008.

Palma-Lazgare, I. R., \& Delgado-Penin, J. A. (2006). HAP-based Broadband Communications under WiMAX Standards - A first approach to physical layer performance assessment. First COST 297 - HAPCOS Workshop, 26-27 October 2006, York, UK.

Papathanassiou, A., Salkintzis, A. K., \& Mathiopoulos, P. T. (2001). A comparison study of the uplink performance of W-CDMA and OFDM for mobile multimedia communications via LEO satellites. Personal Communications, IEEE [see also IEEE Wireless Communications], 8(3), 35-43.

Rappaport, T. S. (1996). Wirless Communications: Principles and Practice. Englewood Cliffs, NJ: Prentice-Hall.

StratXX. (2008). StratXX near space technology. from http:/ / www.lindstrand.co.uk

Technology Review. (2003, Feb.). 10 Emerging Technologies That Will Change the World. Technology Review 106, 33-49.

Thornton, J., Grace, D., Capstick, M. H., \& Tozer, T. C. (2003). Optimizing an Array of Antennas for Cellular Coverage from a High Altitude Platform. IEEE Transactions on Wireless Communications, 2, No. 3, 484-492.

Tozer, T. C., \& Grace, D. (2001). High-Altitude Platforms for Wireless Communications. IEE Electronics and Communications Engineering Journal, 13(3), 127-137.

Vincent, P. J., Tummala, M., \& McEachen, J. (2006, April 2006). An Energy-Efficient Approach for Information Transfer from Distributed Wireless Sensor Systems. IEEE/SMC International Conference on System of System Engineering, Los Angeles, CA, USA.

Yang, Z., Mohammed, A., Hult, T., \& Grace, D. (2007). Assessment of Coexistence Performance for WiMAX Broadband in High Altitude Platform Cellular System and Multiple-Operator Terrestrial Deployments. Paper presented at the 4th IEEE International Symposium on Wireless Communication Systems (ISWCS'07), Trondheim, Norway. 


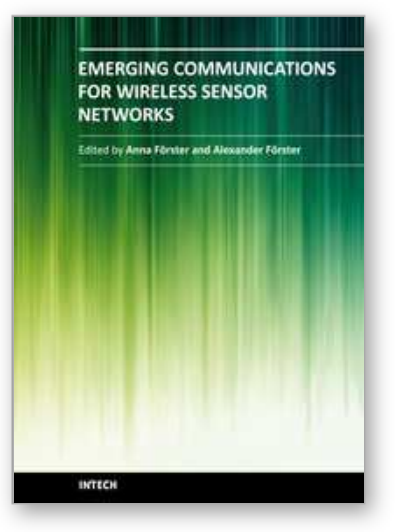

\author{
Emerging Communications for Wireless Sensor Networks \\ Edited by
}

ISBN 978-953-307-082-7

Hard cover, 270 pages

Publisher InTech

Published online 07, February, 2011

Published in print edition February, 2011

Wireless sensor networks are deployed in a rapidly increasing number of arenas, with uses ranging from healthcare monitoring to industrial and environmental safety, as well as new ubiquitous computing devices that are becoming ever more pervasive in our interconnected society. This book presents a range of exciting developments in software communication technologies including some novel applications, such as in high altitude systems, ground heat exchangers and body sensor networks. Authors from leading institutions on four continents present their latest findings in the spirit of exchanging information and stimulating discussion in the WSN community worldwide.

\title{
How to reference
}

In order to correctly reference this scholarly work, feel free to copy and paste the following:

Zhe Yang and Abbas Mohammed (2011). Wireless Sensor Networks Applications via High Altitude Systems, Emerging Communications for Wireless Sensor Networks, (Ed.), ISBN: 978-953-307-082-7, InTech, Available from: http://www.intechopen.com/books/emerging-communications-for-wireless-sensor-networks/wirelesssensor-networks-applications-via-high-altitude-systems

\section{INTECH}

open science | open minds

\author{
InTech Europe \\ University Campus STeP Ri \\ Slavka Krautzeka 83/A \\ 51000 Rijeka, Croatia \\ Phone: +385 (51) 770447 \\ Fax: +385 (51) 686166 \\ www.intechopen.com
}

\author{
InTech China \\ Unit 405, Office Block, Hotel Equatorial Shanghai \\ No.65, Yan An Road (West), Shanghai, 200040, China \\ 中国上海市延安西路65号上海国际贵都大饭店办公楼 405 单元 \\ Phone: +86-21-62489820 \\ Fax: +86-21-62489821
}


(C) 2011 The Author(s). Licensee IntechOpen. This chapter is distributed under the terms of the Creative Commons Attribution-NonCommercialShareAlike-3.0 License, which permits use, distribution and reproduction for non-commercial purposes, provided the original is properly cited and derivative works building on this content are distributed under the same license. 